Published in final edited form as:

Ann Epidemiol. 2011 August ; 21(8): 572-579. doi:10.1016/j.annepidem.2011.04.006.

\title{
Socioeconomic indicators and the risk of acute coronary heart disease events; comparison of population-based data from the United States and Finland
}

\author{
Anna M Kucharska-Newton, PhD MPH(1), Kennet Harald, MA ${ }^{(2)}$, Wayne D Rosamond, \\ PhD ${ }^{(1)}$, Kathryn M Rose, PhD ${ }^{(1)}$, Thomas D Rea, MD MPH ${ }^{(3)}$, and Veikko Salomaa, MD \\ $\mathrm{PhD}^{(2)}$
}

(1)University of North Carolina at Chapel Hill, Chapel Hill, USA (2)National Institute for Health and Welfare, Helsinki, Finland (3)University of Washington, Seattle, USA

\begin{abstract}
Purpose-We wished to determine whether a gradient of association of low socioeconomic status with incidence of coronary heart disease was present in two population-based cohorts, one from United States the other from Finland.

Methods-Using data from the Atherosclerosis Risk in Communities (ARIC) cohort and the Finnish FINRISK cohort, we estimated, with Cox proportional hazard regression models, incidence of sudden cardiac death (SCD), non-sudden cardiac death (NSCD), and non-fatal myocardial infarction (NFMI) for strata of income and education (follow-up: 1987 - 2001). In both cohorts, incidence rates of the three outcomes increased across all SES exposure categories.

Results-Low education was associated with increased hazard of NFMI in both cohorts and with increased risk of SCD among ARIC women. Low income was significantly associated with increased hazard of all three outcomes among ARIC women and with increased hazard of cardiac death among ARIC men. In FINRISK, low income was significantly associated with increased risk of SCD only. Risk of SCD in the low income categories was similar for both cohorts. Smoking, alcohol consumption, and race (ARIC only) did not appreciably alter effect estimates in either cohort.
\end{abstract}

Conclusion-Indices of low SES show similar associations with increased risk of cardiac events in Finland and in United States.

\section{Keywords}

sudden cardiac death; non-fatal MI; socioeconomic factors; cohort studies

(C) 2011 Elsevier Inc. All rights reserved

*Corresponding author: Anna Kucharska-Newton, PhD, MPH Cardiovascular Disease Program Department of Epidemiology University of North Carolina at Chapel Hill, 137 E. Franklin St., Suite 306, Chapel Hill, NC 27514 phone: (919) 9664564 Fax: (919) 9669800 Anna_Newton@unc.edu.

Publisher's Disclaimer: This is a PDF file of an unedited manuscript that has been accepted for publication. As a service to our customers we are providing this early version of the manuscript. The manuscript will undergo copyediting, typesetting, and review of the resulting proof before it is published in its final citable form. Please note that during the production process errors may be discovered which could affect the content, and all legal disclaimers that apply to the journal pertain. 


\section{INTRODUCTION}

Despite clinical and public health interventions, up to fifty percent of total coronary heart disease (CHD) mortality $(1,2)$ continues to be attributed to sudden cardiac death. Identification of those at risk for sudden cardiac death is hampered by lack of specificity of the biological risk factors (3).

Numerous studies have pointed to the association of socioeconomic indicators with sudden cardiac death. Mortality rates for sudden cardiac death, as for other cardiovascular events, show consistent and robust socioeconomic gradients, with higher rates observed among those with higher levels of social deprivation (4). However, the extent to which attributes of socioeconomic status (SES) influence the risk of sudden cardiac death in comparison with the risk of other, nonfatal, cardiovascular outcomes has not been examined extensively.

Gradients in mortality associated with measures of SES have varied across Europe, but persist regardless of overall wealth $(5,6)$. Comparisons of these associations between United States and Europe have not been well studied. The present study was undertaken to substantiate or refute the hypothesis that the association of low SES with the risk of CHD, specifically with the risk of sudden cardiac death, is lower in countries with universal, publicly funded health care services, as compared to the United States where majority of health care insurance is private. We chose to examine the patterns of CHD risk in relation to SES in United States and Finland. The two countries have similar economic growth patterns (7), but differ substantially on issues of social benefits, including access to health care. Our analyses are based on two population-based cohorts: the Atherosclerosis Risk in Communities (ARIC) study and a Finnish population-based cardiovascular risk factor monitoring study, the National FINRISK study.

\section{METHODS}

\section{Study populations}

The ARIC Study-The ARIC study cohort was established in 1987 as a probability sample of 15792 men and women, aged 45 to 64 years, from four communities in the United States: Minnesota, North Carolina, Maryland, and Mississippi (8). Extensive physical examinations were performed at baseline and at three triennial visits. Ongoing follow-up of the ARIC cohort is conducted through annual telephone interviews and surveillance of mortality and cardiovascular morbidity. Follow-up for this study was conducted through December 31, 2001.

The National FINRISK Study-The National FINRISK study consists of a series of population based surveys conducted every five years since 1972 among a stratified random sample of men and women aged 25-64 years, from four regions of Finland (North Karelia, Kuopio, Turku/Loimaa and Helsinki) (9). At the time of each survey, participants are asked to complete a health behavior questionnaire and undergo a personal health examination. Follow-up for mortality and cardiovascular morbidity is conducted through the Finnish Causes-of-Death Register and the Hospital Discharge Register. Cardiovascular diagnoses for those registries have recently been validated (10) and completeness of follow-up is 100 percent for deaths and hospitalizations occurring in Finland.

The present study includes survey participants from the years 1987, 1992 and 1997 with follow-up through December 31, 2001. To assure comparability of results between the FINRISK and ARIC studies, we restrict participants to the 45-64 year age group. 
We excluded from both cohorts, participants with missing information on exposure variables, those with a baseline diagnosis of CHD, and from the ARIC cohort those who reported race other than black or white and small number $(n=55)$ of black participants from the Minnesota and Maryland communities. The total sample size was 13,688 for the ARIC cohort and 9,816 for the FINRISK cohort.

Both studies comply with the Declaration of Helsinki and have been approved by Institutional Review Boards. All study participants signed informed consent forms.

\section{Exposure ascertainment}

Education-ARIC study participants were asked during the baseline interview about their highest attained level of education. Education was then classified as: less than $8^{\text {th }}$ grade, $8^{\text {th }}$ $11^{\text {th }}$ grade, high school or general equivalence diploma, some vocational school, 1-3 years of college, 4 years of college completed and some graduate or professional school. In this study, we defined education as a dichotomous variable with "high school or more education" constituting the reference category and "less than high school education" as the exposure category.

In FINRISK, data concerning participants' highest attained education are obtained by linking the person's unique personal identifier number with information from Statistics Finland. In this study, education was defined as a dichotomous variable with the reference category of at least 10 years of education and the exposure category defined as " 9 years of education or less".

\section{Income}

At baseline interview, ARIC study participants were asked to classify their total family income into one of the following eight categories: $<\$ 5000 ; \$ 5000-\$ 7999 ; \$ 8000-\$ 11999$; \$12 000-\$15 999; \$16 000-24 999; \$25 000-34 999; \$35 000-\$49 000; $\geq \$ 50000$. We collapsed these categories into three groups: less than $\$ 15$ 999; $\$ 16000$ to 34 999; and equal to or greater than 35000 .

In FINRISK, taxable income data were obtained from Statistics Finland. Household income was classified as low, middle and high, according to survey-year specific tertiles of distribution (11).

\section{Outcome classification}

Sudden cardiac death-In the ARIC study, all deaths attributed to heart disease in the main adjudication were reviewed by a separate physician panel to ascertain whether the death was characterized as a sudden, pulseless condition without a known non-cardiac cause. Adjudication was performed for both in- and out-of-hospital deaths on the basis of data obtained from death certificates, informant interviews, physician questionnaires, coroner reports, or hospital discharge summaries. Inter-reviewer reliability was evaluated in a subset of cases. The kappa coefficient describing the classification of sudden cardiac death between the two physician reviewers was 0.79 . Initial discordant classification was resolved by a third independent review. In the FINRISK study, sudden cardiac death is defined as sudden, unexpected cardiac death that occurred out-of-hospital or in the emergency room (12). In the National Causes-of-Death Register, for deaths which occurred outside of the hospital, ICD-9 codes 410-414 and 798, and ICD-10 codes I20-I25, I46 and R96 were taken as sudden cardiac deaths. In a recent comparison with the FINAMI-register, the sensitivity of these diagnoses for fatal coronary events varied between $92 \%$ and $96 \%$, and the positive predictive value varied between $92 \%$ and $97 \%$ [13]. In $60.5 \%(\mathrm{n}=118)$ of the sudden cardiac deaths of the FINRISK component of the present study, the diagnosis was based on autopsy. 
Non-sudden cardiac death-In both ARIC and FINRISK, non-sudden cardiac death was identified during follow-up based on informant interviews, physician and coroner questionnaires, review of medical records and death certificate information and classified as fatal myocardial infarction (MI) or definite or possible fatal CHD that was not identified as sudden cardiac death.

\section{Non-fatal myocardial infarction}

Non-fatal MI was identified during follow-up from the baseline examination through December 31, 2001. In ARIC, this was based on community hospital surveillance, annual questionnaires, or at follow-up examinations. In FINRISK, suspected MI events were identified on the basis of hospital discharge diagnoses in the National Hospital Discharge Register. ICD-9 codes 410 and 411.0, and ICD-10 codes I20.0 and I21-I22 were classified as nonfatal MIs. In both studies non-fatal MI was defined as a definite or probable MI that did not result in death within 28 days (13).

\section{Baseline covariate measurements}

All covariates were measured at baseline unless stated otherwise. Alcohol consumption was based on self-report of beer, wine and liquor consumption. Participants were classified as current, former and never smokers. Cigarette years of smoking were defined as average number of cigarettes smoked per day times number of years smoked. Body mass index was calculated as the ratio of measured weight in kilograms to the square of height in meters. In the ARIC study, total plasma cholesterol levels were determined enzymatically (14) using a Cobas-Bio analyzer with reagents purchased from Boehringer Mannheim Biochemicals, Indianapolis, IN. Baseline diabetes was defined as either a self-reported physician's diagnosis of diabetes, use of hypoglycemic medications, non-fasting serum glucose levels greater than $11.1 \mathrm{mmol} / \mathrm{L}$, or fasting ( $\geq 8 \mathrm{hr}$ ) serum glucose level equal to or greater than 7.0 $\mathrm{mmol} / \mathrm{L}$. Sitting blood pressure was measured three times using a random zero sphygmomanometer. Blood pressure calculations were made as an average of the second and third measurement. In the FINRISK study, serum total cholesterol was measured from fresh serum samples using an enzymatic method (CHOD-PAP, Boehringer Mannheim). Diabetes was defined as either a self-reported physician's diagnosis of diabetes or on the basis of current use of diabetes medication. Blood pressure was measured with a $42 \mathrm{~cm}$ cuff bladder from the right arm of the subject, seated for five minutes prior to the measurement.

\section{Statistical analysis}

Pairwise t-tests and Pearson's $\chi^{2}$ coefficients were used to compare means and proportions of baseline characteristics by education and income. Cox proportional hazard regression of the association of SES and incidence of sudden cardiac death, non-sudden cardiac death, or non-fatal MI was performed with age, race, study center and gender as covariates. Cigarette smoking status, cigarette years of smoking, and exercise were evaluated as potential confounders. In the ARIC study, we used a race/center variable encompassing 5 categories of race and ARIC center.

We evaluated whether the risk of fatal and non-fatal CHD events differed by race (ARIC study only) and gender using partial likelihood ratio tests based on nested models with and without multiplicative interaction terms. Cox proportional hazard assumptions were examined for all variables individually and for the final model using the Cox test, the $\ln -\ln$ plots, and plots of scaled Schoenfeld residuals. Since we evaluated associations of SES with the risk of coronary heart disease independently for the ARIC and FINRISK studies, we could not perform formal statistical comparisons of effect estimates obtained from the two cohorts. All analyses were conducted using STATA 10.0 (College Station, TX) and SAS 9.1 (SAS Institute, Cary, NC). 


\section{RESULTS}

We observed similar age and gender distributions in the ARIC and FINRISK cohorts (Table 1). The proportion of persons with low education was greater in FINRISK as compared to ARIC. In analysis stratified by baseline education and income status, diabetes, BMI, total cholesterol, and age showed similar distributions in both cohorts, with higher levels among those with lower educational attainment and income levels (Table 2). In both cohorts, we observed a higher prevalence of current smoking among those with lower education and a decrease in the prevalence of current smoking across the gradient of median household income. The proportion of never smokers was consistently higher in the FINRISK study as compared to the ARIC study. Likewise, the proportion of individuals with hypertension was consistently higher in the FINRISK study as compared to the ARIC study across categories of both education and income.

In the ARIC cohort, we observed 714 cases of non-fatal MI, 120 cases of non-sudden cardiac death, and 195 cases of sudden cardiac death in the follow up from 1987, through December 31, 2001. During the same follow-up period, we observed 342 cases of non-fatal MI, 66 cases of non-sudden cardiac death, and 148 cases of sudden cardiac death in the FINRISK cohort. Overall incidence rates of non-fatal MI, non-sudden cardiac death, and sudden cardiac death were similar in the two cohorts (Tables 3A and 3B). In both studies, the age-adjusted rates of non-fatal MI were significantly higher than the rates of sudden cardiac death and non-sudden cardiac death, overall and by exposure categories. The incidence rates for all outcomes varied inversely with SES exposure categories.

In the ARIC study, we observed statistically significant interaction between income and gender for non-fatal MI $(\mathrm{p}<0.01)$ and between education and gender for sudden cardiac death $(\mathrm{p}=0.02)$. Those interactions were not significant for non-sudden cardiac death; however, for comparative purposes we stratified all analyses by gender. In the FINRISK study no interaction with gender was observed, therefore risk estimates are presented for the total sample population.

Results of regression analyses for the FINRISK and ARIC cohorts are presented in Figure 1. In both studies, we observed inverse gradients of association of levels of education and income with fatal and non-fatal CHD outcomes. Low education conferred an increased risk of non-fatal MI in both cohorts, but was associated significantly with an increased risk of non-sudden cardiac death and sudden cardiac death only among women (but not men) of the ARIC cohort. In both the ARIC and FINRISK studies we also observed an inverse gradient of association of median household income with non-fatal MI and with sudden and nonsudden cardiac death, although not all associations were statistically significant. In both cohorts the association of low income with the risk of sudden cardiac death was statistically significant. The association of low income with the risk of non-fatal MI was significant only among ARIC women. In the ARIC study, low income was associated significantly with the risk of non-sudden cardiac death for both men and women. In contrast, in the FINRISK cohort, the association of low income with non-sudden cardiac death was modest and not statistically significant.

Since sudden cardiac death was defined in the FINRISK cohort as out-of-hospital cardiac death, in sensitivity analyses we applied the same definition to ARIC data. Our results were comparable to those obtained using the classification of sudden cardiac death that was based on adjudicated review of medical records. We explored the effect of race on the observed associations by limiting the analyses in the ARIC cohort to whites only. Hazard ratios associated with income and education gradients were similar to those for the full cohort for non-fatal MI and sudden cardiac death. Estimates for non-sudden cardiac death were higher 
in analysis limited to whites; however, the small sample size contributed to wide confidence intervals precluding meaningful interpretation of results. Smoking and alcohol consumption did not appreciably change any of the effect estimates for either the ARIC or the FINRISK study.

\section{DISCUSSION}

In this comparative analysis of data from population-based cohorts from the United States and Finland, we observed similar gradients of fatal and non-fatal CHD associated with education and income. Similarities between the two cohorts were most apparent in the association of low education with increased risk of non-fatal MI and in the association of low income with increased risk of sudden cardiac death.

Finland and United States are similar in their rate of economic growth (7). However, they differ profoundly with respect to organization of health care and structure of social services. The Gini coefficient, a measure of economic inequality, is 0.27 for Finland and 0.41 for the United States (a lower Gini coefficient is associated with more equal wealth distribution). Our study aim was to evaluate the presence of gradients of CHD across strata of socioeconomic indicators in population-based cohorts drawn from the two countries. We found that gradients in the incidence of fatal and non-fatal CHD events across education and income categories were present in both the ARIC and FINRISK cohorts.

Previous studies have shown geographic and socioeconomic variation in cardiovascular disease morbidity and overall cardiovascular disease mortality $(15,16)$, however comparatively little information is available concerning the effect of SES on the incidence of sudden cardiac death. Data from existing studies suggest that black race, lower educational attainment, and low neighborhood SES are associated with increased incidence of sudden cardiac death (17-21), or even with increased relative risk of sudden as compared to nonsudden cardiac death (22). Differences in cardiovascular risk profiles $(23,24)$, differential access to care and emergency medical services, as well as differences in levels of psychosocial stress (24) have been suggested as factors contributing to the association of low SES with increased risk of sudden cardiac death. Our data, suggest that factors responsible for increased incidence of sudden cardiac death among the least wealthy, or those with lower educational attainment, operate similarly in different social and political environments. In both studies, diabetes, obesity, high total plasma cholesterol levels, hypertension, and cigarette smoking, all demonstrated inverse gradients with SES. As these variables may be intermediates in the pathways defining the association of income and education with coronary events, we were not able to provide an unbiased assessment of their role in the examined associations. Adjustment for education, smoking, and alcohol consumption did not appreciably alter the estimate of the effect of low income on the risk of CHD events.

This study had several limitations. The ARIC and FINRISK studies differed in the education and income categories. Low education was defined as "less than high school" in the ARIC study and it was "9 years of school or less" in the FINRISK study. These classifications may suggest significant differences in the distribution based on differences in cutpoints. However, the ninth grade of the comprehensive school in Finland is completed by age 16 and constitutes the equivalent of the eleventh grade of schooling in the United States (25). Therefore, education categories in the two study cohorts were comparable. We performed a sensitivity analysis, which used the $9^{\text {th }}$ grade of schooling as the cutpoint between low and high education for the ARIC cohort. Results obtained with these new educational categories were not different from those obtained with education defined as "less than high school". Household income was also defined differently in ARIC and FINRISK. A system of specific 
cutpoints was established for the ARIC study to characterize household income categories (26). In FINRISK, income categories were relative and defined as tertiles of distribution which were established at enrollment. The FINRISK study enrolls participants at five-year intervals and income categories were established for individual enrollment periods. Measures of SES at an aggregate level were not available for the FINRISK study; thus, we could not compare the effects of neighborhood socioeconomic conditions on the risk of fatal as compared to non-fatal CHD events. An additional limitation of this study was the small number of non-sudden cardiac deaths, which was not sufficient for subgroup analyses. Although recruitment into both the ARIC study and the FINRISK study was designed to ensure representativeness, the studies were limited to selected geographical locations within the countries and thus, the findings may not be generalizable to the entire populations of United States and Finland respectively. In the ARIC study, included minorities are limited to African Americans, with no representation of Hispanic groups. Thus, study findings are not generalizable to the fast growing Hispanic populations in the United States.

In conclusion, our analysis of the effect of low income and low education on the incidence of fatal and non-fatal CHD events in cohorts from two distinct countries, suggests that low SES contributes to the risk of CHD events despite differences in social and political environments that may impact access to health care and preventive services in different ways. Extending these analyses to a larger group of socially and politically diverse countries would allow for generalization of our findings.

\section{Acknowledgments}

The Atherosclerosis Risk in Communities Study is carried out as a collaborative study supported by National Heart, Lung, and Blood Institute contracts N01-HC-55015, N01-HC-55016, N01-HC-55018, N01-HC-55019, N01HC-55020, N01-HC-55021, and N01-HC-55022. This work was also supported by a National Research Service Award training grant T32 H2-0007055 (AMK-N).

The authors thank the staff and participants of the ARIC study and the FINRISK study for their important contributions.

\section{Abbreviations}

$\begin{array}{ll}\text { CHD } & \text { coronary heart disease } \\ \text { SES } & \text { socioeconomic status } \\ \text { SCD } & \text { sudden cardiac death } \\ \text { NSCD } & \text { non-sudden cardiac death } \\ \text { NFMI } & \text { non-fatal myocardial infarction } \\ \text { MI } & \text { myocardial infarction } \\ \text { ARIC } & \text { Atherosclerosis Risk in Communities } \\ \text { ICD } & \text { International Classification of Diseases } \\ \text { BMI } & \text { body mass index }\end{array}$

\section{REFERENCES}

1. Zipes DP, Wellnes HJJ. Sudden cardiac death. Circulation. 1998; 98:2334-2351. [PubMed: 9826323]

2. Sotoodehnia N, Zivin A, Bardy GH, Siscovick DS. Reducing mortality from sudden cardiac death in the community: lessons from epidemiology and clinical applications research. Cardiovascular Research. 2001; 50(2):197-209. [PubMed: 11334823] 
3. Kannel WB, Schatzkin A. Sudden death: lessons from subsets in population studies. Journal of the American College of Cardiology. 1985; 5:141B-149B.

4. Hemingway H, Malik M, Marmot M. Social and psychosocial influences on sudden cardiac death, ventricular arrhythmia and cardiac autonomic function. European Heart Journal. 2001; 22(13): 1082-1101. [PubMed: 11428849]

5. Mackenbach JP, Stirbu I, Roskam AJ, Schaap MM, Menvielle G, Leinsalu M, et al. Socioeconomic inequalities in health in 22 European countries. N Engl J Med. 2008; 358(23):2468-81. [PubMed: 18525043]

6. Palosuo H, Koskinen S, Lahelma E, Kostiainen E, Prättälä R, Martelin T, et al. Health Disparities in Finland. Trends in Socioeconomic Health Differences. 2009

7. International Monetary Fund. 2007. cited; Available from: http://www.imf.org/external/pubs/ft/weo/2009/01/weodata/weorept.aspx?sy=2007\&ey=2014\&sc $\mathrm{sm}=1 \& \mathrm{ssd}=1 \&$ sort=country \&ds=.\&br=1\&pr1.x=62\&pr1.y=13\&c=172\%2C111\&s=NGDP_R\%2 CNGDPRPC \%2CNGDPDPC\%2CLUR\&grp=0\&a=

8. The ARIC Investigators. The Atherosclerosis Risk in Community (ARIC) Study. Design and Objectives. Am. J. Epidemiol. 1989; 129(4):687-702. [PubMed: 2646917]

9. Vartiainen E, Puska P, Pekkanen J, Toumilehto J, Jousilahti P. Changes in risk factors explain changes in mortality from ischaemic heart disease in Finland. BMJ. 1994; 309(6946):23-27. [PubMed: 8044063]

10. Pajunen, Pa; Koukkunen, Hb; Ketonen, Mc; Jerkkola, Td; Immonen-Raiha, Pe; Karja-Koskenkari, Pf, et al. The validity of the Finnish Hospital Discharge Register and Causes of Death Register data on coronary heart disease. European Journal of Cardiovascular Prevention \& Rehabilitation. 2005; 12(2):132-137. [PubMed: 15785298]

11. Salomaa V, Miettinen H, Niemela M, Ketonen M, Mahonen M, Immonen-Raiha P, et al. Relation of socioeconomic position to the case fatality, prognosis and treatment of myocardial infarction events; the FINMONICA MI Register Study. J Epidemiol Community Health. 2001; 55(7):47582. [PubMed: 11413176]

12. Salomaa V, Ketonen M, Koukkunen H, Immonen-Raiha P, Jerkkola T, Karja-Koskenkari P, et al. Decline in Out-of-Hospital Coronary Heart Disease Deaths Has Contributed the Main Part to the Overall Decline in Coronary Heart Disease Mortality Rates Among Persons 35 to 64 Years of Age in Finland. The FINAMI Study Circulation. 2003; 108(6):691-696.

13. White AD, Rosamond WD, Chambless LE, Thomas N, Conwill D, Cooper LS, et al. Sex and race differences in short-term prognosis after acute coronary heart disease events: the Atherosclerosis Risk In Communities (ARIC) study. Am Heart J. 1999; 138(3 Pt 1):540-8. [PubMed: 10467206]

14. Siedel J, Hagele EO, Ziegenhorn J, Wahlefeld AW. Reagent for the enzymatic determination of serum total cholesterol with improved lipolytic efficiency. Clin Chem. 1983; 29(6):1075-80. [PubMed: 6851096]

15. Marmot M. Health in an unequal world: social circumstances, biology and disease. Clinical Medicine, Journal of the Royal College of Physicians. 2006; 6:559-572.

16. Diez Roux AV, Merkin SS, Arnett D, Chambless L, Massing M, Nieto FJ, et al. Neighborhood of residence and incidence of coronary heart disease. N Engl J Med. 2001; 345(2):99-106. [PubMed: 11450679]

17. Reinier K, Stecker EC, Vickers C, Gunson K, Jui J, Chugh SS. Incidence of sudden cardiac arrest is higher in areas of low socioeconomic status: A prospective two year study in a large United States community. Resuscitation. 2006; 70:186-192. [PubMed: 16814445]

18. Soo L, Huff N, Gray D, Hampton JR. Geographical distribution of cardiac arrest in Nottinghamshire. Resuscitation. 2001; 48(2):137-147. [PubMed: 11426475]

19. Escobedo LG, Caspersen CJ. Risk factors for sudden coronary death in the United States. Epidemiology. 1997; 8:175-180. [PubMed: 9229210]

20. Nichol G, Thomas E, Callaway CW, Hedges J, Powell JL, Aufderheide TP, et al. Regional variation in out-of-hospital cardiac arrest incidence and outcome. JAMA. 2008; 300(12):1423-31. [PubMed: 18812533] 
21. Galea S, Blaney S, Nandi A, Silverman R, Vlahov D, Foltin G, et al. Explaining Racial Disparities in Incidence of and Survival from Out-of-Hospital Cardiac Arrest. Am. J. Epidemiol. 2007; 166(5):534-543. [PubMed: 17584756]

22. Escobedo LG, Zack MM. Comparison of sudden and nonsudden coronary deaths in the United States. Circulation. 1996; 93(11):2033-6. [PubMed: 8640979]

23. Hinkle LE Jr. Thaler HT, Merke DP, Renier-Berg D, Morton NE. The risk factors for arrhythmic death in a sample of men followed for 20 years. Am J Epidemiol. 1988; 127(3):500-15. [PubMed: 3341356]

24. Lynch JW, Kaplan GA, Cohen RD, Tuomilehto J, Salonen JT. Do Cardiovascular Risk Factors Explain the Relation between Socioeconomic Status, Risk of All-Cause Mortality, Cardiovascular Mortality, and Acute Myocardial Infarction? Am. J. Epidemiol. 1996; 144(10):934-942. [PubMed: 8916504]

25. Hopkins JD. The Educational System in Finland. Background, Structure, Equivalencies, and New Directions. World Education News and Reviews. 1990; 4:10-16.

26. Diez-Roux AV, Nieto FJ, Tyroler HA, Crum LD, Szklo M. Social Inequalities and Atherosclerosis The Atherosclerosis Risk in Communities Study. Am. J. Epidemiol. 1995; 141(10):960-972. [PubMed: 7741126] 

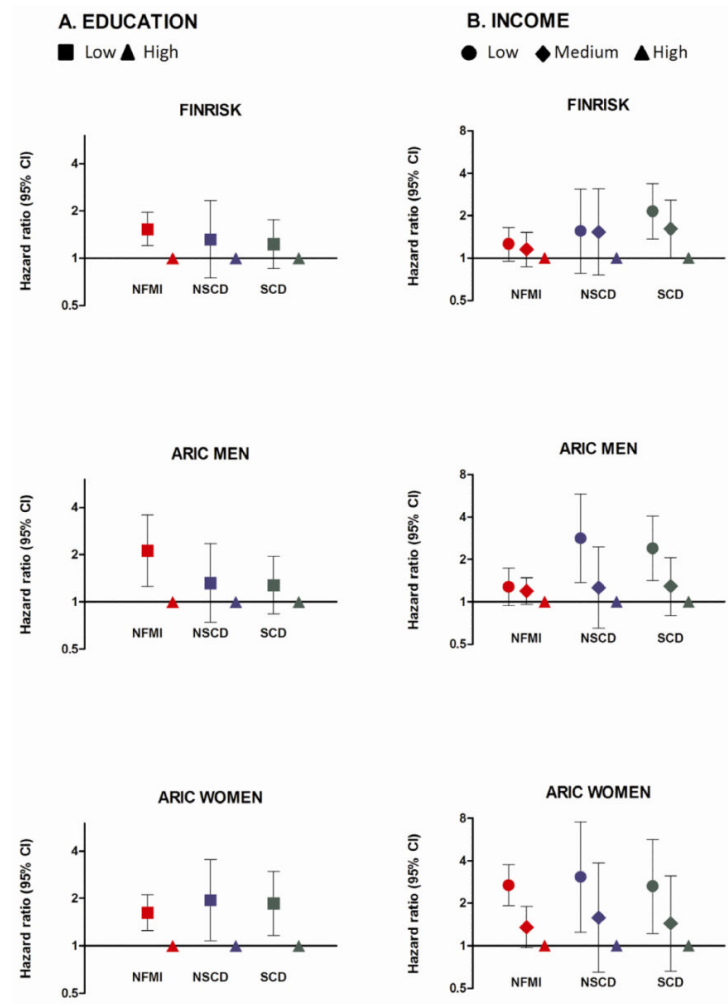

Figure 1.

Association of education (A) and income (B) with the risk of sudden cardiac death (SCD), non-sudden cardiac death (NSCD), and non-fatal MI (NFMI); the ARIC and FINRISK cohorts 1987-2001. Hazard ratios (95\% confidence intervals) adjusted for age, gender (FINRISK only), and race (ARIC only). 
Table 1

Baseline characteristics. Comparison of the ARIC and FINRISK studies

\begin{tabular}{lcc}
\hline Characteristic & ARIC (n=13 712) & FINRISK (n=9816) \\
\hline Age, years (SD) & $54.0(5.7)$ & $54.2(5.8)$ \\
Gender, \% Male & 43.6 & 47.4 \\
Race, \% Black & 25.9 & - \\
Education, \% & & \\
low & 22.8 & 55.7 \\
high & 77.2 & 44.3 \\
Income, \% & & \\
low & 22.6 & 33.0 \\
middle & 32.7 & 33.3 \\
high & 44.7 & 33.7 \\
Smoking & & \\
\% current & 26.0 & 21.7 \\
$\%$ former & 31.5 & 21.3 \\
$\%$ never & 42.5 & 57.0 \\
Alcohol consumption (g/day) & $6.1(14.0)$ & $6.8(13.2)$ \\
Hypertension, \% & 34.2 & 60.8 \\
BMI & $27.6(5.3)$ & $27.6(4.5)$ \\
Total cholesterol (mmol/L) & $5.6(1.1)$ & $6.1(0.4)$ \\
Diabetes (\% yes) & 11.1 & 6.3 \\
\hline
\end{tabular}

Low education was defined as 9 years or less of education in the FINRISK study and less than high school in the ARIC study.

Combined household income was categorized according to tertiles of distribution in the FINRISK study. In the ARIC study, income was classified into categories of low, medium and high according to 1987 earning levels with the cutpoints at \$15 999 and \$34 999 . 


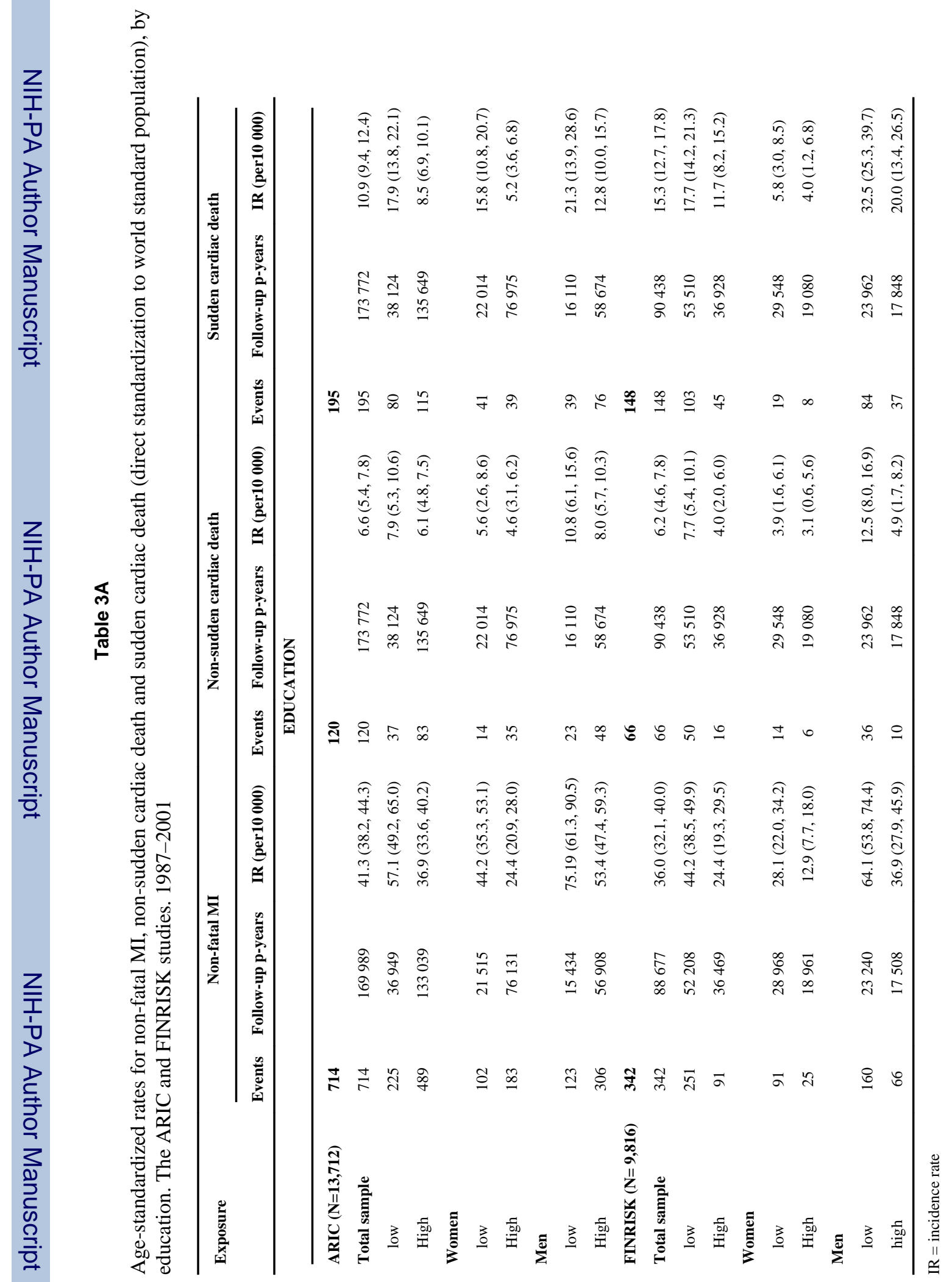




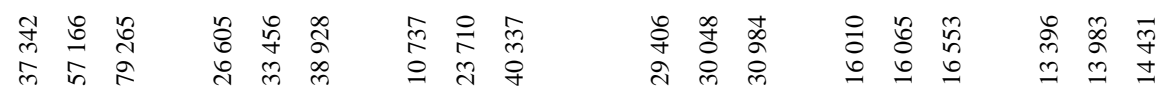

$\infty$ in

in 용

సి

$\stackrel{x}{\infty}$

$\infty \simeq$

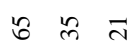

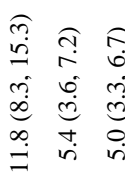

$\stackrel{\circ}{\circ} \sigma$

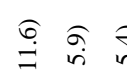

i $9 \infty$

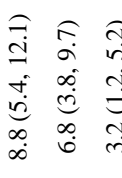

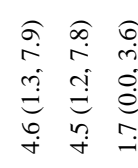

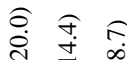

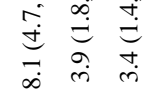

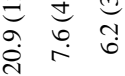

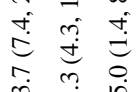

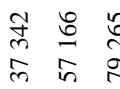

요요

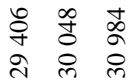

$\begin{array}{lll}\circ & 0 & n \\ 0 & 8 & n \\ 0 & 0 & 0\end{array}$

ळ

g

$\operatorname{9}$

เุ

$\bar{m} \pi$

$a \infty$

$4 \simeq a$

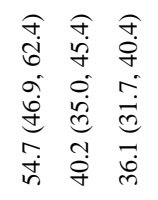

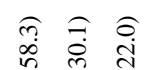

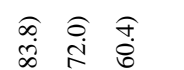

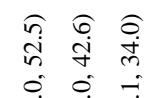

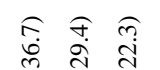

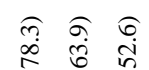

ฮั

흉

$\cong$

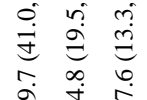

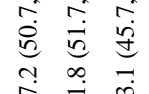

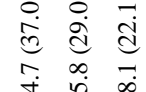

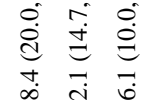

$\therefore \therefore$

तै

j

है

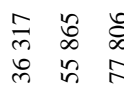

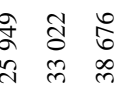

$\begin{array}{lll}0 & 0 \\ 0 & + & 0 \\ 0 & 1 & 0\end{array}$

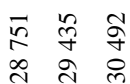

तิ

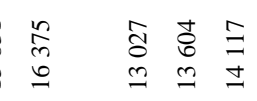

$\exists \cong$

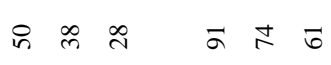

